\title{
Run-and-tumble particles with hydrodynamics: sedimentation, trapping and upstream swimming
}

\author{
R. W. Nash ${ }^{1}$, R. Adhikari ${ }^{1,2}$, J. Tailleur ${ }^{1}$ and M. E. Cates ${ }^{1}$ \\ ${ }^{1}$ SUPA, School of Physics, University of Edinburgh, \\ JCMB Kings Buildings, Edinburgh EH9 3JZ, United Kingdom \\ ${ }^{2}$ The Institute of Mathematical Sciences, CIT Campus, Chennai 600113, India
}

(Dated: October 25, 2018)

\begin{abstract}
We simulate by lattice Boltzmann the nonequilibrium steady states of run-and-tumble particles (inspired by a minimal model of bacteria), interacting by far-field hydrodynamics, subject to confinement. Under gravity, hydrodynamic interactions barely perturb the steady state found without them, but for particles in a harmonic trap such a state is quite changed if the run length is larger than the confinement length: a self-assembled pump is formed. Particles likewise confined in a narrow channel show a generic upstream flux in Poiseuille flow: chiral swimming is not required.
\end{abstract}

PACS numbers: 47.63.Gd, 87.10.Mn, 87.17.Jj

The motility of microorganisms raises basic physics questions that range from local swimming mechanisms [1] to many-body emergent phenomena 4, 5, 7]. In the latter context, even grossly simplified models represent a challenging and active area of nonequilibrium statistical mechanics [4. In some cases experimental nearcounterparts to these models can be devised in which various complicating factors (cell division, chemotaxis, etc.) are environmentally or genetically suppressed [8.

Indeed certain bacteria, including $E$. coli, exhibit motion which can be idealized as a 'run-and-tumble' model. Here straight 'runs' at constant speed $v$ are punctuated by sudden, rapid and complete randomizations in direction, or 'tumbles', occurring stochastically with rate $\alpha$ [8. The mean run length is $\ell=v / \alpha$ and duration $1 / \alpha$; at larger length and time scales Fick's law is obeyed, with diffusivity $D=v^{2} / d \alpha$ in $d$ dimensions [9]. This model offers an important paradigm for a diffusion process that is fundamentally non-Brownian. Subtle consequences of this are manifest for particles in external force fields, such as gravity or a harmonic trap [10]. In the first case, the gravitational decay length $\lambda$ falls strictly to zero when the gravitational force $f$ exceeds the propulsive force $f_{p}$, in contrast to Brownian particles for which $\lambda=D / f$ [10]. In a harmonic trap $(f=-k r)$, particles are strictly confined within a radius $r^{*}=f_{p} / k$; and for $\ell \gtrsim r^{*}$ the maximum density occurs at $r \sim r^{*}$ not $r=0$. In this limit, a particle in the trap interior rapidly swims out to $r^{*}$ and stays there a long time until its next tumble [10].

The qualitative physics of the aforementioned results is robust to both a distribution in $v$, or a residual true Brownian diffusivity. On the other hand, because there is no underlying free energy (which would give a Boltzmann distribution as the unique steady state), long-range hydrodynamic interactions (HI) between the particles could have major consequences, even for steady-state behavior. Several computational approaches to address hydrodynamics have been developed [5, but none have addressed the basic physics problems considered below: (a) sedimentation in a container with a solid bottom; (b) confinement by a harmonic trap; and (c) Poiseuille flow between parallel plates. These we consider at small but finite particle density, so that in (a,b) only the far-field hydrodynamics are important. In (c), the main hydrodynamic effect is instead the coupling to an imposed flow.

Problems of bacteria in force fields may appear to have little direct relevance to biology [8]. This could explain a surprising lack of experiments on both bacterial sedimentation, e.g., by centrifugation, and trapping (where the regime $\ell \gtrsim r^{*}$ might be achieved using recent optical methods 11.) We argue that these simple cases demand to be understood before one can claim to explain more complex (and biologically relevant) ones, and hope our work will stimulate new experiments to help fill such gaps.

In this Letter we address run-and-tumble systems confined by gravity, traps or walls. In the first two cases, our goal is to see whether the nonequilibrium steadystates found without HI [10] survive with HI present. For sedimentation, we find only weak effects of HI. In contrast, for a harmonic trap, we find that only for $\ell \ll r^{*}$ is the near-Gaussian distribution seen without HI maintained; whenever $\ell \gtrsim r *$ the 'density-inverted' steady state is destroyed by HI, replaced instead by a remarkable selfassembled pump-like structure. We explore in detail the origin of this instability, in which the local co-alignment of swimmers causes HI to add coherently rather than with random signs, vastly enhancing their effects. Thirdly, we address swimmers confined between walls at separations $h$. HI do not prevent swimmers from accumulating at the walls [7, where a weak Poiseuille flow causes strong upstream alignment, and hence, for $h \lesssim \ell$ a net upstream particle flux. This mechanism is much simpler than one explored previously at larger $h[2$.

An obstacle to simulations is the expense of handling near-field aspects of the HI [5]. These are clearly important at high enough density, but depend on a swimmer's precise geometry and stroke. In contrast, the far-field flow around a swimmer, in the absence of any body force acting on it, is of universal stresslet form [5, with force dipole strength $s=\nu f_{p} a$. Here $a$ is a hydrodynamic radius, and $\nu$ is order one; the organism is 'extensile' for $\nu>0$, 'contractile' for $\nu<0$. (Most bacteria are exten- 
sile.) At low concentrations, modeling only the stresslet fields should capture any universal macroscopic consequences of HI. As shown below, this simplification drastically reduces the computational cost.

Method: We use the lattice Boltzmann (LB) method to handle the fluid momentum transport [12, coupled to a description of each swimmer as a pair of off-lattice point particles at fixed separation $\nu a$ (we choose $\nu=2$ ). At these points, forces $\pm \mathbf{f}_{p}$ (of fixed magnitude, with + denoting the 'head' of the swimmer) are exerted on the fluid, creating an extensile stresslet. These forces are mapped onto the LB lattice by an optimal discretization [13, alongside any external force $\mathbf{f}$ which we assume to act only on the head. (This force is passed directly onto the fluid: there is no particle inertia.) A local fluid velocity (excluding a self-term) is then interpolated from the lattice to the head particle [13; the swimmer co-rotates with the local fluid and moves relative to it with an additional velocity $\mathbf{u}=\left(\mathbf{f}_{p}+\mathbf{f}\right) / 6 \pi \eta a$. Although pointlike for forcing purposes, our particles have finite hydrodynamic radius, $a=0.05$; particle scale Reynolds numbers are small, as required $\left(5 \times 10^{-3}\right)$. As shown in [13] our method efficiently simulates dilute forced colloids, using $a$ values much smaller than the lattice spacing (unity). Indeed with $a=0.05$ we have simulated sedimentation of $2^{16}=65536$ swimmers at volume fraction $\phi \sim 6.5 \times 10^{-5}$, on a lattice of size $128^{2} \times 32$, using a serial code. In contrast, previously published works using fully resolved algorithms are limited to at most a few hundred particles [5, 6]. A fully resolved LB study of the same system would involve $a$, and thus $L$, about 20 times larger [13. This would require use of very large parallel computers. With the latter, we hope in future to use our far-field code to address situations involving millions of particles.

Sedimentation: We now turn to our results, first briefly outlining those for sedimentation. (An additional figure and further discussion are available in appendix A.) We have computed steady state density profiles $\rho(z)$ under a constant gravity force $f$, at various $w \equiv f / f_{p}$, in both small and large systems (1000 and 65536 particles respectively), with hard walls at the base and ceiling and periodic boundary conditions horizontally in both cases. Far from a proximal region (of height $\sim \ell$ for $w \ll 1$, in which the density is strongly perturbed by the basal wall) our numerical density profiles closely resemble the analytic result of [10] (exact in an unbounded domain without HI): $\rho(z) \sim \exp [-z / \lambda]$ with $\lambda(w) \rightarrow 0$ as $w \rightarrow 1$. Indeed, the exponential form is maintained (within error) with HI present, and $\lambda(w)$ has for small $w$ a very similar form to that without HI. However as $w \rightarrow 1, \lambda$ seemingly remains higher than predicted (see appendix A). Nonetheless, the role of HI in altering the steady state remains modest: in particular, we see no evidence of HI inducing macroscopic flow patterns (which, in the absence of a free energy, would be possible in principle). This strongly contrasts with our results for traps, to which we turn next.

Traps: Fig 1 shows steady state densities $\rho(r)$ for par-

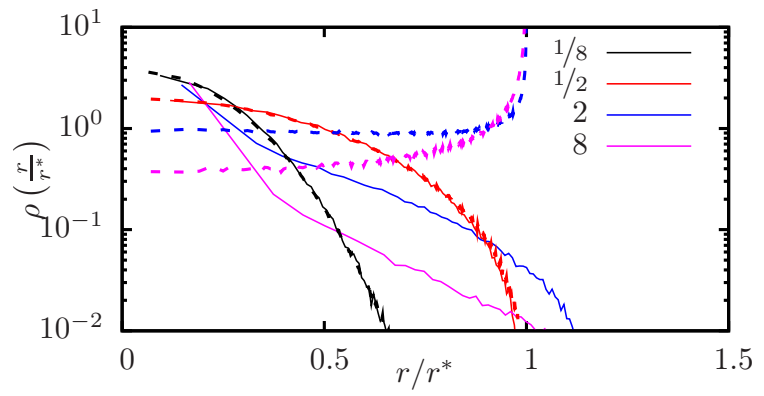

FIG. 1: (Color online) Steady state density for particles confined to harmonic traps. Solid lines: LB simulations; dashed lines: numerics of [10]. Key: $\zeta=\ell / r *$ values. Note that without HI particles cannot exceed the trap radius $r^{*}$.

ticles confined to harmonic traps with various ratios of run length to confinement length, $\zeta=\ell / r^{*}$. These are compared to the numerics of [10] without HI; the latter were also used as the initial condition for our runs. For small $\zeta$, we see very little effect of HI: both algorithms show the Gaussian distributions expected for particles of diffusivity $D$ in a harmonic trap. For larger $\zeta$ however, HI dramatically destabilize the 'density-inverted' state (with $\rho(r)$ maximal at $r^{*}$ ) that arises without HI. Rather than approaching a steady state with zero macroscopic flux (as the non-HI system does), the system moves to an attractor in which rotational symmetry is broken by the formation of a swarm of outward-swimming particles. To a good approximation the swarm remains stationary at a radius $r_{s}<r^{*}$, where the propulsive force balances the collective drag and the trapping force. The latter is passed on to the fluid to create a macroscopic flow: one has in effect a self-assembled pump. The resulting flow is mainly of stokeslet form, with fluid flowing opposite to the swimming direction. (A slow rotation of this direction, which might cancel the stokeslet term on time averaging, is detectable, but the rotation rate vanishes as $\alpha \rightarrow 0$.) When a swimmer in the clump tumbles, it is ejected in a random direction. The velocity gradient of the stokeslet flow rotates it to point upstream; confined by the trap, it eventually must rejoin the swarm. A series of snapshots is given in Fig 2, and a movie in [14.

This emergent structure contains regions of high density whose details will depend on near-field physics that we do not resolve. However, the initial instability is well captured by far-field hydrodynamics. The end-state is likewise robust, in that any instability leading to formation of a single oriented swarm within the confines of a trap (harmonic or otherwise) will cause a similar 'self-pumping' state. To better understand the initial instability, we consider the limit of infrequent tumbling, $\ell / r^{*} \gg 1$. Here, without HI, the unique steady state comprises a thin uniform layer of outward swimmers at $r^{*}$, each with its propulsive force balanced by the external trapping force [10]. Coupling this to a solvent, we have locally a two dimensional layer of particles exerting inward point forces (stokeslets) on the fluid. 

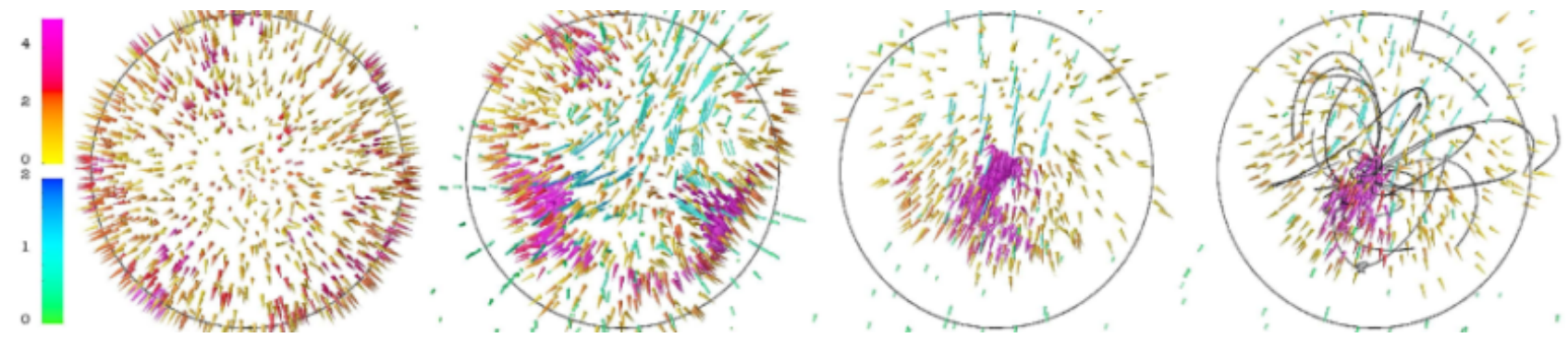

FIG. 2: (Color online) Simulation of $10^{3}$ swimmers in a harmonic trap. The trap radius is indicated by the circle; $\zeta=8$. Swimmers are marked with cones and shaded by local density in units of the mean (key: upper color bar). For the final image, randomly selected particles' trajectories over the preceding time interval $\Delta t=0.5 \alpha^{-1}$ are shown. Arrows depict fluid velocity colored by magnitude in units of the swim speed $v$ (key: lower color bar). For a movie see supplementary material [14].

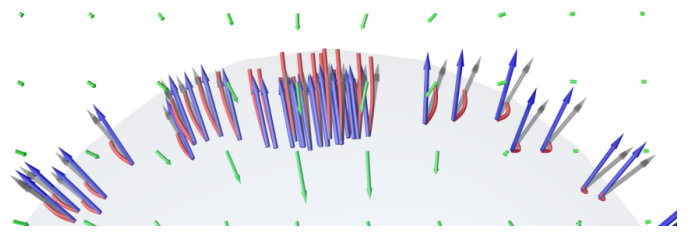

FIG. 3: (Color online) Snapshot showing the early stage instability in a simulation of 1000 swimmers, initially uniformly and randomly distributed on the surface of a trap. Green arrows: local flow field. Red lines: swimmer trajectories. Blue arrows: swimming direction. Grey arrows: radial direction. Swimmers in the dense patch (centre) move radially inwards. Those either side are initially advected away from the patch, but rotate their swimming direction towards it.

Because the particles on the initial shell can have not only tangential displacements (causing density changes) but also radial and orientational ones, a formal stability analysis of the coupled hydrodynamics of the swimmers in this layer is not practicable. (Moreover, noise in the run and tumble dynamics could alter the conclusions.) Nonetheless, by a careful series of simulations (detailed, with additional figures, in appendix B) we have identified that the instability mode is caused initially by tangential density fluctuations on the surface of the trap $\left(r=r^{*}\right)$. Such density fluctuations are inevitable if the total number of swimmers is not infinite, and lead to instability via the following mechanism. Any surface patch that happens to be denser than the surrounding ones will generate locally an excess stokeslet-like flow (not cancelled by the contributions from distant parts of the shell). This flow has two effects. First, it advects the swimmers in the dense patch towards the center of the trap; second, it rotates neighboring particles so that these start swimming towards the dense patch, creating positive feedback (Fig. 3). The resulting clump creates a macroscopic flow that sweeps the remaining particles towards itself (Fig. 2).

On the other hand, if in our simulations we radially displace a patch without altering its density, the feedback is negative; this also applies for a patch in which only the orientation of the swimmers is perturbed. Thus, although radial and orientational modes are strongly excited in the subsequent dynamics, they are not the cause of the initial instability (see appendix B). Notably the same instability is present for contractile rather than extensile swimmers [15. This is fully consistent with the mechanism above, in which the stokeslet from the confining force is transferred to the fluid by a stationary swimmer (the sign of whose stresslet is then less important).

Since the densest initial patch usually outstrips its competitors to create a dominant stokeslet, there is little excitation of modes above the first spherical harmonic. (This can also be explained by expanding in such harmonics the flow arising from a nonuniform shell of point forces [15.) The instability reported here is somewhat related to that found by Crowley, who showed that dense regions in a sedimenting suspension fall more rapidly [16, with the fastest growth at long wavelengths. However, the initial force balance is different enough to prevent a direct mapping from one instability to the other.

Upstream swimming: We turn finally to run-andtumble particles confined between parallel plates at separation $h$. In the absence of HI, we expect the majority of particles to be near the walls whenever $\ell \gtrsim h$. The reason is the same as for the spherical trap, but the HI-induced instability of the layer found in that case is suppressed here by the no-slip boundary condition at the wall.

The dynamics of a swimmer adjacent to a wall can be complex 2, 3, 8. Instead of resolving the near-field HI we apply a truncated Lennard Jones (LJ) potential (range 0.25 lattice sites) which balances the normal component of the propulsive force. The tangential component still leads to motion. In practice, near-field HI could reduce or enhance this motion and also can, for chiral swimstrokes, lead to circular orbits [3. Treating these complexities would introduce nonuniversal parameters into the model; by neglecting them, we can clarify whether or not they are essential in the context of Poiseuille flow. Here upstream swimming has been observed experimentally, and a detailed mechanism proposed that requires chiral swimming [2]. Without questioning this result for the regimes addressed in 2, we note that for narrow micro-channels $(\ell \gtrsim h)$, a much simpler mechanism is also 


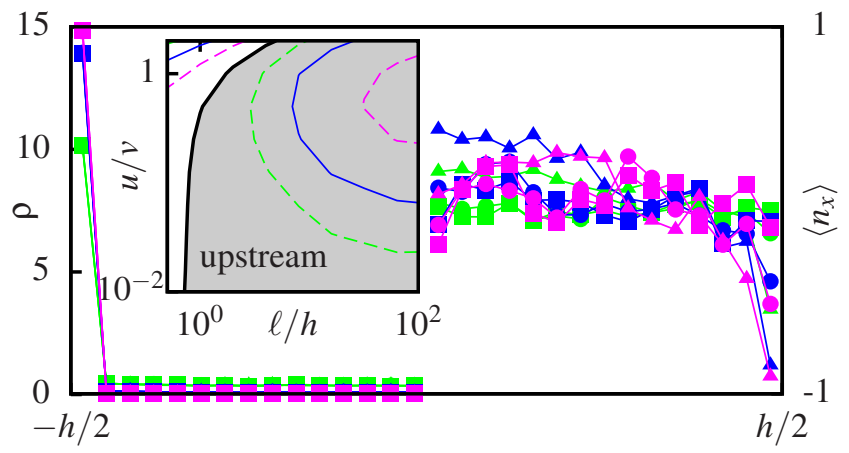

FIG. 4: (Color online) Main figure: density (left) and orientation (right) profiles. Parameters: $u / v=1 / 2,1 / 16,1 / 128$ (triangles, circles, squares) and $\ell / h=2,16,128$ (green, blue, pink). Inset: state diagram showing contours of the average swimmer speed, at interval $v / 4$. Starting from $+0.5 v$ in the top left (solid blue), the value decreases to zero (thick black), further reducing to $-0.75 v$ on the right (dashed pink). At the wall, particles feel a truncated LJ potential of range 0.25 .

at work. Recall that in this regime, any swimmer in the bulk of the channel moves to the wall layer and stays there a long time before tumbling. On approach to the wall any such swimmer is rotated by the velocity gradient so as to align its swimming direction upstream. This effect is strong whenever the product of the wall shear rate and the transit time across the gap is large; this equates to $u / v \gtrsim 1$ with $u$ the flow speed at the mid-plane. This condition ensures that for a uniform $\rho(\ell \ll h)$ the upstream wall flux is outweighed by a downstream bulk flux. However for $\ell \gtrsim h, \rho$ is peaked at the walls, and a net upstream flux results. For typical particle trajectories see appendix D, Fig 4 shows the region in parameter space where there is a net upstream flux, and plots of the particle density and mean orientation in this region. These results are slightly shifted quantitatively, but not qualitatively, by increasing the LB resolution, or adopting slightly different (but still inelastic [10]) wall collision rules.

Conclusion: Above we have presented results from an efficient hydrodynamic simulation of dilute run-andtumble swimmers. Under gravity we find only perturbative effects of $\mathrm{HI}$ on the flux-free steady state of 10 . Much stronger effects are found in geometries where, without HI, all particles are swimming locally in the same direction. This result is consistent with a crude power counting argument: for a sheet of swimmers, the $1 / r^{2}$ velocity contributions from each stresslet cannot give a large effect unless they add coherently. This happens for the trap and the parallel plate geometry, but only when the run-length exceeds the confinement length. For harmonic traps, the flux-free steady state is then replaced by one in which a symmetry-breaking swarm of swimmers acts as a pump. For swimmers in microchannels, outward-oriented particles at the confining walls are aligned by shear to create an upstream particle flux without the need for chiral motion. We hope

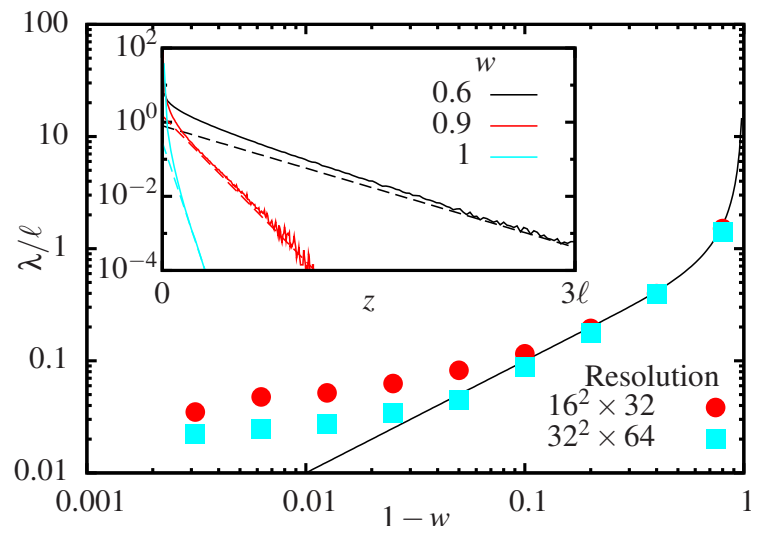

FIG. 5: (Color online) Main figure: $\lambda / \ell$ vs. $1-w$ from fits to the distal part of $\rho(z)$ in a system of 1000 particles. Solid line: prediction of 10]; circles, squares: simulations with HI at two resolutions. Inset: profiles of the swimmer density with the fitted exponentials (dashed). For better statistics, we repeated the runs for $w=0.9,0.95,0.975$ in a $128^{2} \times 32$ system with $2^{16}=65536$ swimmers and found extremely similar $\lambda$ values. We checked that for $w \geq 1$ the profile collapses entirely if we switch off $\mathrm{HI}$ after its formation.

that these predictions will stimulate new quantitative experiments on the fundamental physics of self-propelled micro-organisms in suspension.

We thank D. Marenduzzo, A. Morozov, K. Stratford and W. Poon for discussions and EPSRC EP/E030173 and EP/H027254 for funding. MEC is funded by the Royal Society.

\section{Appendix A: Results for sedimentation}

Fig 5 shows results for steady state density profiles $\rho(z)$ under a constant gravity force $f$, at various $w \equiv f / f_{p}$, in a system of 1000 particles with hard walls at the base and ceiling and periodic boundary conditions horizontally. As discussed in the main text, far from a proximal region the exponential form predicted without $\mathrm{HI}$ in 10 is maintained (within error) with HI present. However as $w \rightarrow 1, \lambda$ seemingly remains higher than predicted. The details depend however on the resolution used (Fig 5) and our studies suggest that the larger fitted $\lambda$ is in fact a proximal effect caused by the random stirring of the fluid by particles near the wall, causing upward advection of some of the particles above. If so, we expect this effect to die out at larger distances with the asymptotic decay length $\lambda$ reverting to its non-HI value. In any case, the role of HI in altering the steady state appears modest: in particular, we see no evidence of $\mathrm{HI}$ inducing macroscopic flow patterns (which, in the absence of a free energy, would be possible). 


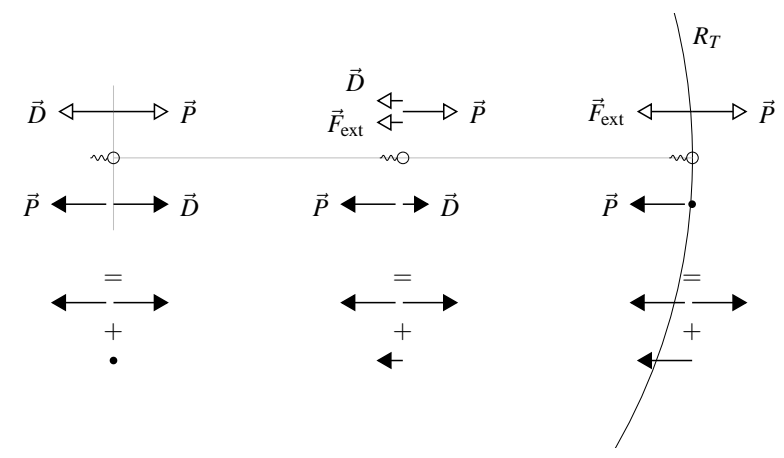

FIG. 6: Forces on a swimmer as function of its distance to the center of the trap. The upper, open-headed arrows show the forces on the swimmer's body and the lower, filled arrows show the forces on the fluid. $P$ is the propulsive force, $D$ the drag and $F_{\text {ext }}$ the external, trapping force. The very bottom arrows decompose the swimmer's effect on the fluid into stokeslet and stresslet components, illustrating that the external, trapping force is effectively passed onto the fluid.

\section{Appendix B: The origin of the trap instability}

In the following we show how the disorder in the position of swimmers on a shell generates the instability, and compare it with other possible mechanisms that are shown to be less relevant. To do so we consider an initial condition where the swimmers have swum as far as they can and are thus located on the surface of a sphere of radius $r^{*}=f_{p} / k$. In the absence of any flow, the only forces acting on the swimmers are their propulsive forces and the trapping force. Because both the fluid and the swimmers are at rest, there is no drag force and the only force felt by the fluid is the propulsive force: effectively the external trapping force has been passed onto the fluid and the effect of the swimmer on the fluid is to create a static, inward pointing stokeslet whose amplitude equals the propulsive force (see Fig. 6).

Because our swimmers are point-like particles at zero Reynolds number, the flow created by $N$ swimmers is simply the superposition of the flow created by each one of them. To understand the effect of such flows on a swimmer, let us consider the simpler situation of an inward pointing stokeslet of magnitude $p$ held fixed at the north pole of a sphere of radius $r^{*}$ and a swimmer located at an Euler angle $\theta, \varphi=0$ on the surface of this sphere (see Fig. 7). The flow field created at this point by the stokeslet is given by [17]

$$
\vec{u}=\frac{p}{16 \pi \eta r^{*} \sqrt{2(1-\cos \theta)}}\left(\begin{array}{c}
\sin \theta \\
0 \\
\cos \theta-3
\end{array}\right)
$$

while the corresponding vorticity is:

$$
\vec{\omega}=\frac{p}{8 \sqrt{2} \pi\left(r^{*}\right)^{2} \eta(1-\cos \theta)^{3 / 2}}\left(\begin{array}{c}
0 \\
-\sin \theta \\
0
\end{array}\right)
$$

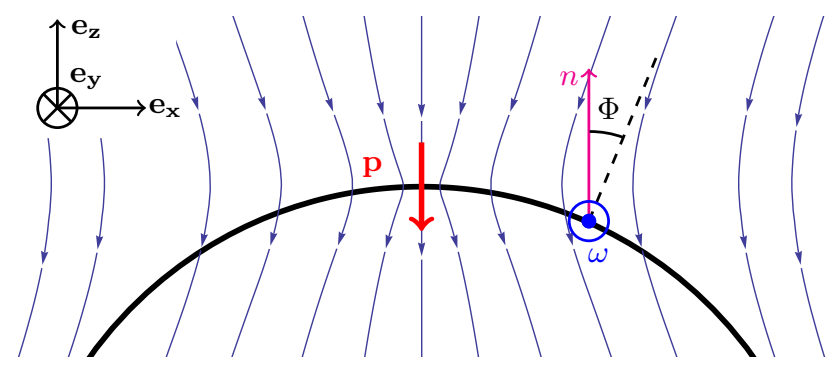

FIG. 7: We consider the flow field generated by a stokeslet $\mathbf{p}$ (red arrow) held fixed at the north pole of a sphere of radius $r^{*}$ and its effect on a swimmer located at Euler angles $\theta$ and $\varphi=0$, whose orientation (magenta arrow) makes an angle $\Phi$ with the normal to the sphere. The vorticity is indicated in blue and tend to rotate the swimmer towards the stokeslet.

For small $\theta$, that is when the swimmer is in the vicinity of the stokeslet, the flow has two effects: the swimmer is advected inwards $\left(u_{z}<0\right)$ and rotated by the vorticity field towards the stokeslet $\left(\omega_{x}=\omega_{z}=0\right.$ while $\omega_{y}<0$, the swimmer rotates counterclockwise on figure 7). As time goes on, the swimmer thus also start swimming towards the stokeslet. This argument can be made more quantitative by considering the dynamics of the swimmer (neglecting the effect of the swimmer on the flow):

$$
\dot{\Phi}=\omega ; \quad \gamma(\dot{\mathbf{r}}-\mathbf{u})=\mathbf{f}_{\mathbf{p}}+\mathbf{f}_{\mathbf{t}}
$$

where $\gamma$ is the effective friction coefficient of the swimmer [15], $\mathbf{f}_{\mathbf{p}}$ the propulsive force of the swimmer and $\mathbf{f}_{\mathbf{t}}$ the trapping force. Let us then introduce $\mathbf{e}_{\mathbf{n}}=$ $\sin \theta \mathbf{e}_{\mathbf{x}}+\cos \theta \mathbf{e}_{\mathbf{z}}$ and $\mathbf{e}_{\theta}=\cos \theta \mathbf{e}_{\mathbf{x}}-\sin \theta \mathbf{e}_{\mathbf{z}}$ the unit vectors normal and tangent to the sphere at the position of the swimmer, so that the position of the swimmer is $\mathbf{r}=r \mathbf{e}_{\mathbf{n}}$. Using the expression of the flow, the equations of motion of the swimmer can be rewritten

$$
\begin{aligned}
\dot{r} & =\frac{p}{16 \pi \eta R \sqrt{2(1-\cos \theta)}}(1-3 \cos \theta)-f \gamma^{-1}(1-\cos \Phi) \\
r \dot{\theta} & =\frac{3 p \sin \theta}{16 \pi \eta R \sqrt{2(1-\cos \theta)}}-\gamma^{-1} f \sin \Phi \\
\dot{\Phi} & =\frac{p \sin \theta}{16 \sqrt{2} \pi \eta R^{2}(1-\cos \theta)^{3 / 2}}
\end{aligned}
$$

Let us now consider the dynamics of a swimmer that is initially pointing out radially $(\Phi=0)$ and is close to the stokeslet, say $\theta \lesssim \pi / 4$. Initially, one finds as expected $\dot{r}<0$ so that the swimmers falls inwards. The second equation show that, initially, the flow advects the swimmer away from the stokeslet $(\dot{\theta} \geq 0$ at $t=0)$. As time goes on however, the angle $\Phi$ increases (third equation) and $\dot{\theta}$ will change sign. At larger time, the swimmer is indeed swimming towards the stokeslet. When the particles move away from the surface of the trap, equations (B4) become more complex and we do not attempt an analytic approach and include these equations only to illustrate the qualitative behaviour of the swimmers. 


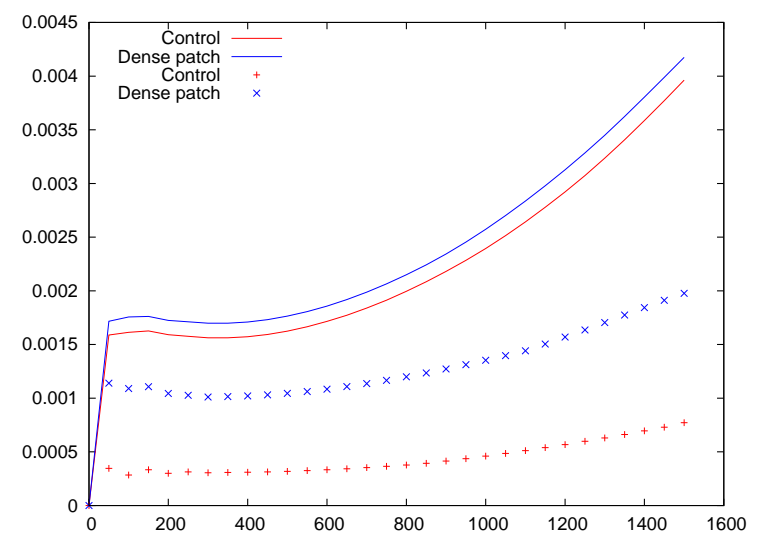

FIG. 8: Comparison of the flow fields generated by a control simulation, starting from a random uniform initial condition, and one with the densest patch on the top. The solid lines correspond to $\left\langle|v|_{\infty}\right\rangle$ and are identical for the two sets of simulations. This is expected since the initial condition with the densest patch on the top aims at being a simple rotation of the uniform one. The discrete symbols correspond to $|\langle v\rangle|_{\infty}$. In this case, the control decreases much more that the simulation with the densest patch on the top which shows that density fluctuation on a scale attained by the random distribution of swimmers suffice to control the instability.

Let us now come back to the problem of $N$ swimmers in the absence of any external flow. Firstly, note that by symmetry one sees that a perfectly uniform layer of stokeslets cannot create any flow and is thus in mechanical equilibrium. Because we consider a finite number of swimmers, the density of stokeslets on the surface on the sphere cannot, however, be uniform but can be decomposed as the sum of two contributions: a perfectly uniform layer of inward pointing stokeslets, representing the average density, and a layer of inward and outward pointing stokeslets representing the more dense and less dense regions, i.e., the density fluctuations. Close to the denser regions, one can thus expect to see the scenario described above for the motion of a swimmer in an inward pointing stokeslet flow. This is indeed what we see in a simulation of 1000 swimmers randomly distributed on the boundary of the trap (see Fig. 3). Around the denser patch located at the top, the far-field flow created by the swimmers resembles that of a stokeslet. [19] Furthermore, the trajectories of the neighboring swimmers resemble the one described above for a single swimmer in a stokeslet flow. Density fluctuations within the surface of the trap are thus a strong candidate for the origin of the instability.

To confirm this role we compare simulations where the densest patch of the initial condition is placed at the top of the trap with those obtained from random uniform initial conditions. Let us first specify what we mean by a dense patch. Since there are 1000 swimmers, every patch of surface $S$ contains on average $N_{s}=250 S /\left[\pi\left(r^{*}\right)^{2}\right]$ swimmers, with fluctuations of the order of $\sqrt{N_{s}}$. We thus choose a patch size $S$ that is a small fraction of the sphere but large enough that fluctuations are not much larger than the mean density. We achieve this by considering a spherical cap defined by $\theta \leq \pi / 12$, which contains on average 17 swimmers. To cover the sphere with patches of this size, one would need roughly 60 patches, which means that the densest patch contains on average 27 swimmers. 20. Our 'dense patch' initial condition is thus constructed as follows: each swimmer is placed uniformly on the sphere with probability $p$ or uniformly on the top spherical cap with probability $1-p$. In practice, $p$ is chosen so that the average number of swimmers in the spherical cap is 27 . We then want to compare the instability between this initial condition and the one where swimmers are uniformly randomly distributed (from now on, we refer to this initial configuration as 'control'). If tangential density fluctuations are not the cause of the instability, the statistical outcome of the 'dense patch' and 'control' simulations should be the same.

To compare the instability, we can look at the generated flow fields. From our lattice Boltzmann simulations we get a $3 \mathrm{D}$ grid with fluid velocities $\mathbf{v}_{i}$ at the nodes. Any measure of the intensity of the flow yields qualitatively similar results and we thus consider $|\mathbf{v}|_{\infty} \equiv \sup _{i}\left|\mathbf{v}_{i}\right|$. In figure 8 we report $\left\langle|\mathbf{v}|_{\infty}\right\rangle$ and $|\langle\mathbf{v}\rangle|_{\infty}$, where the averages are done over 50 runs both for the control and the dense patch cases. We see that the two initial conditions give very similar $\left\langle|\mathbf{v}|_{\infty}\right\rangle$ : this validates our 'dense patch' initial condition which is not very different, once rotated appropriately, from a 'control' one. Since the random uniform case is perfectly isotropic, we expect the flow field to vanish when averaged over many runs. When comparing $|\langle\mathbf{v}\rangle|_{\infty}$, we indeed see that such is the case for the control; by increasing further the number of runs over which the average is done, the non-zero residual flow would continue to decrease. For the dense patch case however, the flow remains much larger. Because the denser patch at the top has to compete with other dense patches, there is some randomness in the orientation of the flow generated by the instability and the intensity of the flow has decreased. It remains however three times larger than the control case, which shows that fluctuations of density of the scale of those observed in the simulations are sufficient to create the instability.

A less quantitative but more direct comparison can be done by starting a control simulation, locating the patch where the instability starts and rotating the view in such a way that it is at the top. One can then compare it with a 'dense patch' run (see figure 9p. Both configuration are indeed very similar, confirming the role of tangential density fluctuations in creating the instability. 

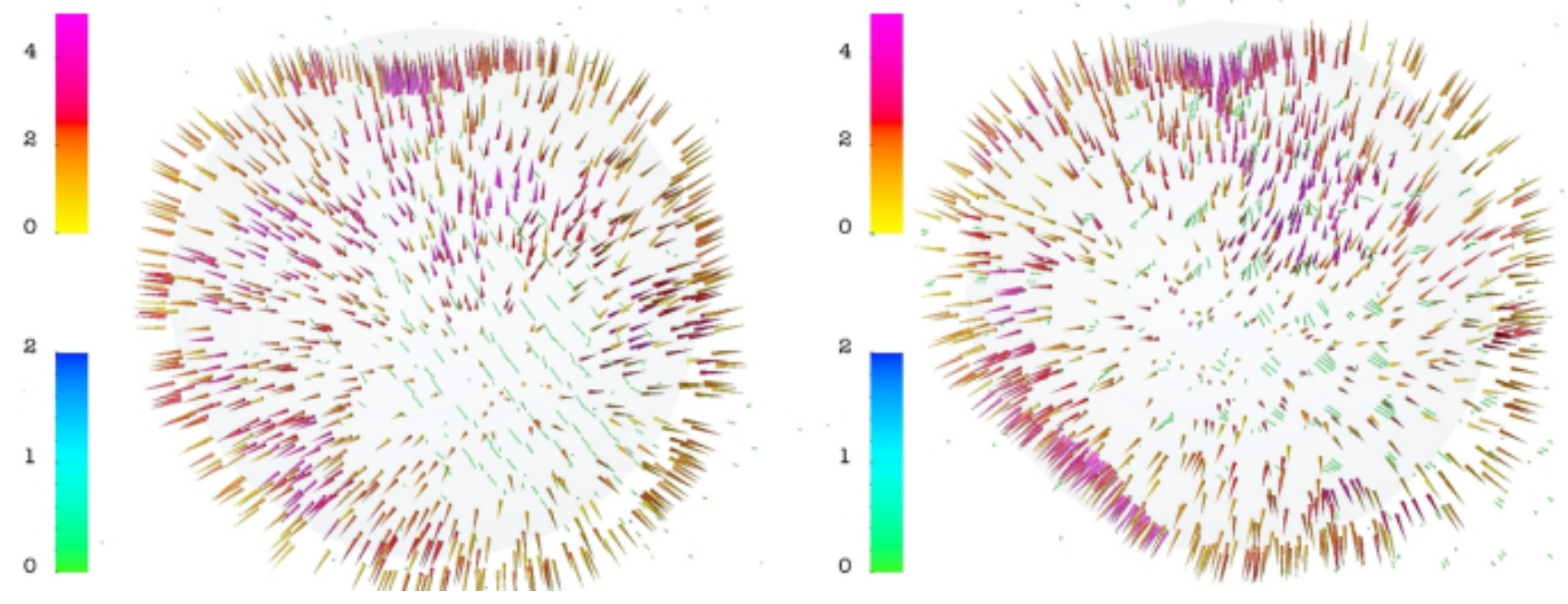

FIG. 9: Comparison between a random uniform initial condition (left) and one where the densest patch is 'seeded' on the top (right). The random uniform one has been rotated so that the instability starts on the top. We see very similar flow patterns and displacement of swimmers close to the north pole. Note that because of secondary dense patches, the rest of the configuration can slightly differ.

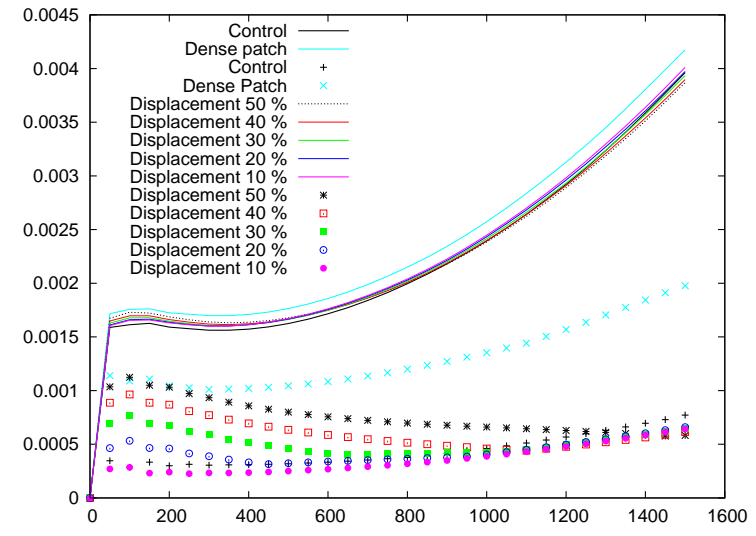

FIG. 10: Comparison between control, dense patch, and radially displaced initial conditions for displacements ranging from $10 \%$ to $50 \%$ of the trap radius. Solid lines correspond to $\left\langle|\mathbf{v}|_{\infty}\right\rangle$ whereas symbols correspond to $|\langle\mathbf{v}\rangle|_{\infty}$. All initial conditions produce similar flow (solid line), and thus similar instability. The averaged flows of the displaced initial conditions and the control are the same at large times, showing that these perturbations have a much smaller effect on the setup than the one due to density fluctuations. Note that the non-zero initial flow is caused by particles swimming back to the border of the trap.

\section{Other type of perturbations}

In addition to the non-uniformity of the density of swimmers on the surface of the trap, other sources of perturbation could be responsible for the instability. First, the swimmers can fluctuate in radial position ('radial dis- order'), and second they may not all be strictly pointing outwards ('angular disorder'). We now show below that these fluctuations are less relevant than the tangential density fluctuations.

\section{a. Radial Displacement}

To study the role of radial displacement, we place the swimmers at random over the whole sphere uniformly and then move downwards those in the same spherical cap as previously contained the dense set of bacteria. (There is no longer an excess density in this cap, however.) Again we compare the instability by looking at the flow fields. As can be seen in figure 10, $\left\langle|\mathbf{v}|_{\infty}\right\rangle$ is left unchanged for displacements up to half the radius of the trap. This means that displacements of swimmers, even much larger those spontaneously observed in simulations, do not seem to generate any kind of instability with a stronger effect than the one seen in their absence. Let us now turn to the comparision of $|\langle\mathbf{v}\rangle|_{\infty}$ to look for systematic effects. At large times, the flow field is the same as the control one and much smaller than that of the dense patch; this perturbation does not fix the direction of the instability. Hence radial disorder, even for large displacements, does not control the instability observed from the random initial configurations.

\section{b. Rotation}

To investigate the effect of disorder in the swimmers orientation, we first place the swimmers at random over the whole surface uniformly and then modify the orien- 


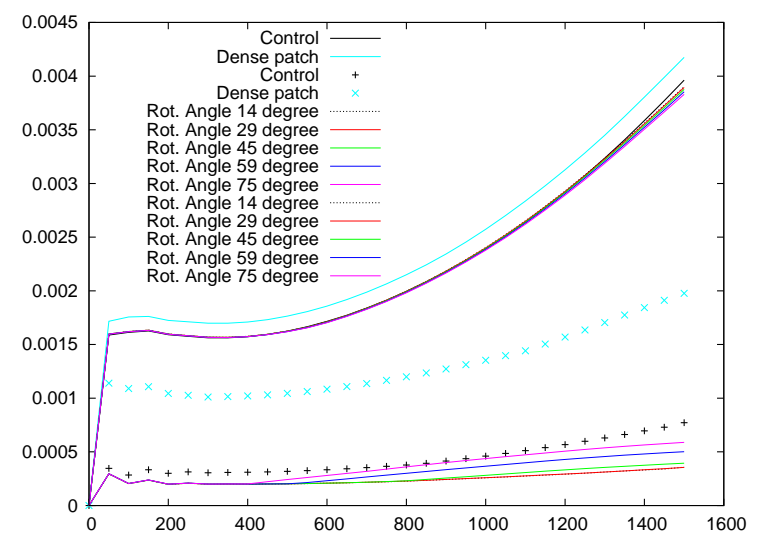

FIG. 11: Comparison between control, dense patch and randomly rotated initial conditions for several rotation angles. Solid lines correspond to $\left\langle|\mathbf{v}|_{\infty}\right\rangle$ whereas symbols correspond to $|\langle\mathbf{v}\rangle|_{\infty}$. All initial conditions produces similar strength of flow, and thus of instability. The average flow of the random rotations equals that of the control, showing that these perturbation have a much smaller effect on the setup than the density fluctuations.

tation of the swimmers that are in the top spherical cap defined by $\theta \leq \pi / 12$. We either generate randomly a rotation matrix and apply it to all these simmwers (uniform rotation) or we pick up one rotation matrix per swimmer (random rotation). The strength of the perturbation is controlled by varying the average rotation angle of the distribution of the rotation matrix. In both cases, $\left\langle|\mathbf{v}|_{\infty}\right\rangle$ perfectly overlaps with the control (see figure 11 and 12, while the quantity $|\langle\mathbf{v}\rangle|_{\infty}$ is as small as the control. These results confirm that the initial rotation has no part to play in triggering the instability.

\section{Appendix C: Brownian Motion}

Interestingly, our stability analysis allows us to evaluate the impact of Brownian motion on the trap instability. For E. coli, thermal diffusivity is $D \simeq$ $0.3 \mu \mathrm{m}^{2} / \mathrm{s}$ whereas its rotational counterpart is $D_{\text {rot }} \simeq$ $0.15 \mathrm{rad}^{2} / \mathrm{s}$ [18. Over the time scale of a second, which is the average time between two tumbles, this corresponds to diffusion length and angle of about half a micrometer and 20 degrees. The simulations presented in the previous subsections shows that these perturbations are negligible when compared to the density fluctuations. Brownian motion should thus not affect the trap instability.

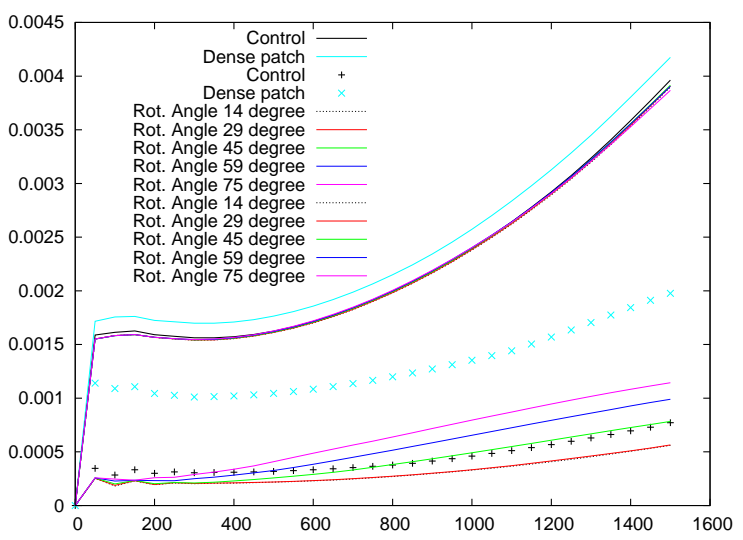

FIG. 12: Comparison between control, dense patch, and uniformly rotated initial conditions for several rotation angles. Solid lines correspond to $\left\langle|\mathbf{v}|_{\infty}\right\rangle$ whereas symbols correspond to $|\langle\mathbf{v}\rangle|_{\infty}$. All initial conditions produce similar strength of flow, and thus of instability. The average flow of the uniformly rotated initial condition is however of the same order as the control one, showing that these perturbation have a much smaller effect on the setup than the density fluctuations.

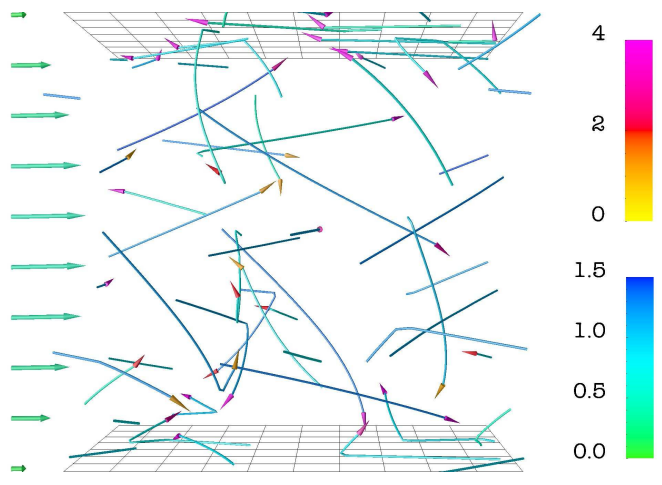

FIG. 13: (Color online) 40 randomly chosen swimmers (of 1000 simulated) in a Poiseuille flow between plates. Swimmers are marked with cones and shaded by relative local density (key: upper color bar). Trajectories over the preceding time interval $\Delta t=0.5 \alpha^{-1}$ are shown, colored by magnitude of lab-frame speed in units of $v$ (key: lower color bar). The flow profile is shown on the left (same key).

\section{Appendix D: Channel Flow}

An additional figure for channel flow, showing swimmer trajectories, is given as Fig. 13.
[1] R. Golestanian and A. Ajdari, Phys. Rev. Lett. 100, 038101 (2008); P. Garstecki and M. Cieplak, J. Phys.
Cond. Mat. 21, 200301 (2009) and references therein. [2] J. Hill et al, Phys. Rev. Lett. 98, 068101 (2007) 
[3] W. R. DiLuzio et al., Nature 435, 1271 (2005); S. van Teeffelen and H. Löwen, Phys. Rev. E 78, 020101(R) (2008).

[4] A. Czirok, A.-L. Barabasi and T. Vicsek, Phys. Rev. Lett. 82, 209-212 (1999); J. Toner, Y. H. Tu and S. Ramaswamy, Annals of Phys. 318, 170-224 (2005); A. Sokolov et al., Phys. Rev. Lett. 98, 158102 (2007).

[5] I. Llopis and I. Pagonabarraga, EPL 75, 999 (2006); C. M. Pooley, G. P. Alexander and J. M. Yeomans, Phys. Rev. Lett. 99, 228103 (2007) T. Ishikawa and T. J. Pedley, Phys. Rev. Lett. 100, 088103 (2008)

[6] Our work is similar in spirit to that of P. T. Underhill, J. P. Hernandez-Ortiz and M. D. Graham, Phys. Rev. Lett. 100, 248101 (2008), and D. Saintillan and M. J. Shelley, Phys. Rev. Lett. 99, 058102 (2007). These both use far-field Green function methods and address several thousand particles. However, we expect our LB-based approach to prove more adaptable in complex geometries and more suited to parallelization [12.

[7] H. H. Wensink and H. Löwen, Phys. Rev. E 78, 031409 (2008).

[8] H. C. Berg, E. coli in Motion, Springer, NY 2004.

[9] M. J. Schnitzer, Phys. Rev. E 48, 2553-2568 (1993).

[10] J. Tailleur and M. E. Cates, Phys. Rev. Lett. 100, 218103
(2008); EPL 86, 60002 (2009).

[11] B. Lincoln et al., Biomed. Microdevices 9 703-710 (2007)

[12] S. Succi, The Lattice Boltzmann Equation for Fluid Dynamics and Beyond, Clarendon, Oxford (2001).

[13] R. W. Nash, R. Adhikari and M. E. Cates, Phys. Rev. E 77, 026709 (2008).

[14] See supplementary material for movies http://prl.aps.org/supplemental/PRL/v104/i25/e258101

[15] R. Nash, PhD Thesis, University of Edinburgh (2009).

[16] J. M. Crowley, Phys. Fluids 19, 1296 (1976); R. Lahiri and S. Ramaswamy, Phys. Rev. Lett. 79, 1150 (1997).

[17] C. Pozrikidis Introduction to theoretical and computational fluid dynamics, (Oxford University Press, Oxford) 1997

[18] S.P. Strong, B. Freedman, W. Bialek, R. Koberle, Phys. Rev. E 57, 4604 (1998)

[19] because the dense patch has a finite size the near field is however slightly different.

[20] This can be checked by negelecting the correlations between the number of swimmers in the different patches coming from the total constrains, and using cumulative generating function of the binomial distribution. 